\title{
«Wir sind die Kompetenzpartner in Gesundheitsfragen - wer denn sonst!»
}

\author{
Seit Anfang 2014 leitet Dr. med. Peter Wiedersheim neu die FMH-Delegiertenver- \\ sammlung. Seinen Einstand als Vorsitzender hat er inzwischen erfolgreich bestan- \\ den: Kompetent führte er Ende Januar durch die Veranstaltung und schaffte es, \\ trotz zahlreicher Traktanden genügend Raum für Austausch und Diskussion zu er- \\ möglichen. Wie sich die FMH-Delegiertenversammlung entwickeln soll und wie er \\ seine Rolle aus Vorsitzender dieses Gremiums sieht, erfahren Sie im nachfolgenden \\ Interview.
}

Interview:

Jacqueline Wettstein

Leiterin Abteilung Kommunikation
Korrespondenz: Jacqueline Wettstein FMH

Abteilung Kommunikation Elfenstrasse 18

CH-3000 Bern 15

Tel. 0313591150

Fax 0313591112

kommunikation@fmh.ch
Seit 2014 sind Sie der neue Vorsitzende der FMH-Delegiertenversammlung. Was hat Sie dazu bewogen, für dieses Amt zu kandidieren?

Einerseits waren es die vielen Kolleginnen und Kollegen, die mich für eine Kandidatur motiviert haben, andererseits ist es sicher auch die spannende und herausfordernde Aufgabe. Dank meiner langjährigen standespolitischen Erfahrung und dem guten Netzwerk sehe ich als Vorsitzender der Delegiertenversammlung auch die Chance, die Basis der Ärzteschaft besser in die Gesundheitspolitik zu integrieren. Ich will zudem auch die Delegiertenversammlung mit der längst fälligen Umsetzung des von der Ärztekammer am 6. Dezember 2007 mit überwältigendem Mehr angenommenen Führungsmodells der FMH weiter optimieren und auch die Wirkung der FMH nach innen und aussen verbessern.

Sie waren während sieben Jahren eines der 33 Mitglieder der Delegiertenversammlung. Wie haben Sie diese Zeit erlebt?

Es war eine sehr spannende und lehrreiche Zeit. Schon in der ersten Sitzung wurde mir der Vorsitz der Arbeitsgruppe «Neue Führungsmodelle für die FMH» übertragen. Es folgten zahlreiche weitere interessante Aufgaben. Die Delegiertenversammlung selbst hat initial eher zaghafte Schritte unternommen, heute ist sie aber weitaus trittsicherer unterwegs. $\mathrm{Zu}$ optimieren ist nicht nur aus meiner Sicht die Verständigung: Nebst gewissen kulturellen Unter-

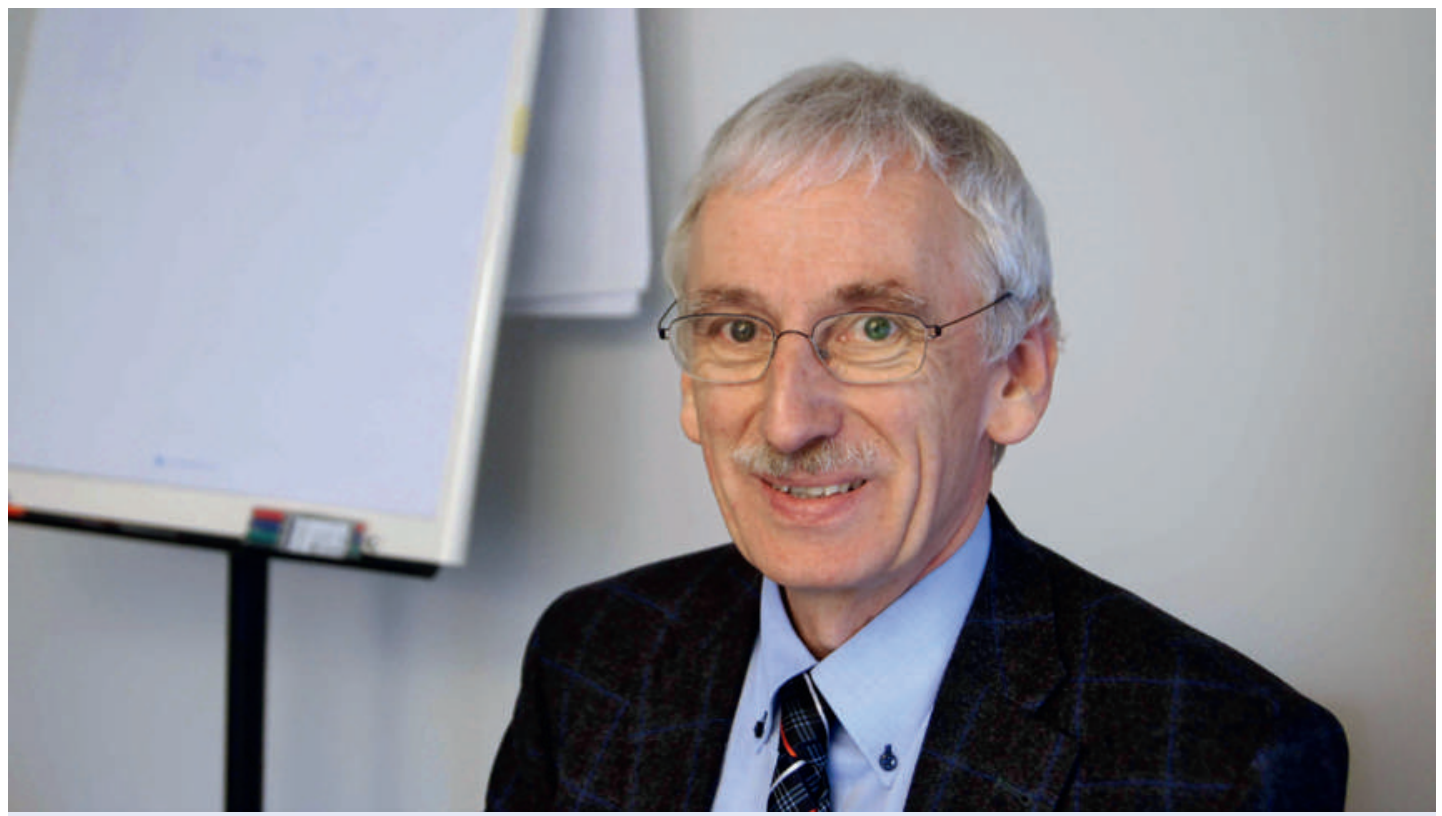

Peter Wiedersheim, der neue Vorsitzende der FMH-Delegiertenversammlung, will die Basis der Ärzteschaft besser in die Gesundheitspolitik integrieren. 


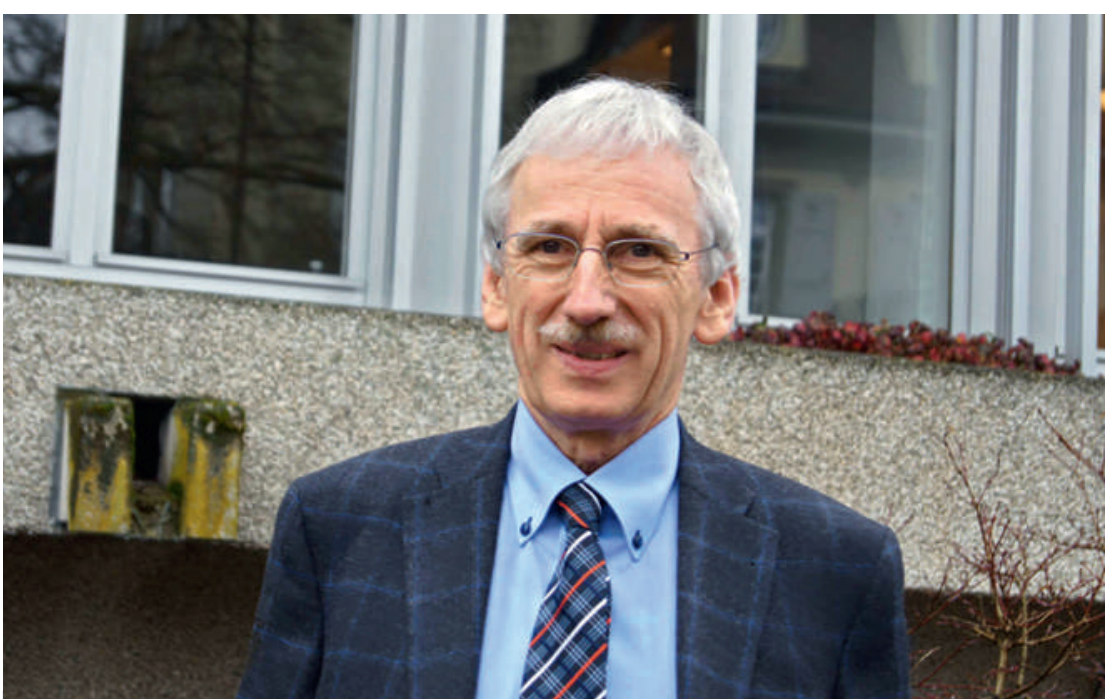

«Als Player im Schweizer Gesundheitswesen müssen wir das Agenda-Setting vorausschauend angehen.»
Welche Themen stehen für Sie in den kommenden Monaten im Fokus?

Kurzfristig sind die Themen weitgehend gesetzt, um nicht zu sagen «diktiert». Bei der Flut von externen Aufgaben kämpfen wir heute viel zu reaktiv - das hat ja vermutlich sogar System. Um als Player zu agieren, müssen wir das Agenda-Setting aktiv und vorausschauend angehen! Meine klaren Favoriten sind den Nutzen für Patienten zu optimieren unter Einbezug der volkswirtschaftlichen Gesamtkosten, das sektorielle Kostendenken zu bekämpfen und noch mehr Transparenz zu schaffen. Ebenso ist es wichtig, eine gute Versorgungsforschung aufzubauen als auch die Qualität der Behandlung und Versorgung weiter zu verbessern. Wer aufhört, besser zu werden, hat aufgehört, gut zu sein! Gerade über unser qualitatives Engagement müssen wir auch mehr und besser kommunizieren - getreu dem Motto: Tue Gutes und sprich darüber!

schieden zwischen der Romandie und der Deutschschweiz resultieren noch zu oft unnötige sprachliche Missverständnisse. Auch im Sinne der gegenseitigen Wertschätzung will ich der optimierten Kommunikation besondere Beachtung schenken.

Was möchten Sie als Präsident der Delegiertenversammlung bewirken?

Ich wünsche mir eine selbstsichere und aktive Ärzteschaft, die sich ihrer wichtigen Rolle und Verantwortung in unserem Gesundheitswesen bewusst ist und hierfür auch Verantwortung übernimmt. Die Gesundheitspolitik der verschiedenen FMH-Gremien soll mehr Wirkung zeigen, sowohl nach innen als auch nach aussen. Ich will die Basis und insbeson-

\section{«Die Ärzteschaft muss selbstbewusster werden, das heisst aber auch mehr Verantwortung übernehmen, und zwar auf allen Stufen».}

dere die jungen Ärztinnen und Ärzte besser in die Führungsebenen unserer FMH einbeziehen. Dazu bedarf es nicht nur eines optimierten Informationsflusses von der Basis zu den Führungsebenen, sondern auch von diesen zur Basis. Es ist mir ein Anliegen, dass die sprachliche und kulturelle Vielfalt der Ärzteschaft auch in der Delegiertenversammlung gelebt wird. Dabei soll nicht vergessen werden, dass wir unsere Anliegen am besten mit einem möglichst geschlossenen Auftreten nach aussen platzieren können. Generell will ich vor allem zu mehr Selbstbewusstsein der Ärzteschaft beitragen: Wir sind die Kompetenzpartner in allen Gesundheitsfragen - wer denn sonst!

\section{«Wer aufhört, besser zu werden, hat aufgehört, gut zu sein!».}

Was wünschen Sie sich von den Mitgliedern der Delegiertenversammlung?

Führungserfahrung, eine gute Integration in die Verbandspolitik und eine optimale, beidseitige Vernetzung mit der Basis sowie den Verbandsgremien - das wären meine Anliegen an meine Kolleginnen und Kollegen. Selbstverständlich freue ich mich auch auf die weiterhin gute kollegiale Teamarbeit.

Peter Wiedersheim wurde Ende 2013 als Nachfolger von Thomas Heuberger zum neuen Vorsitzenden der FMH-Delegiertenversammlung gewählt. 1953 geboren, legte er 1978 sein Staatsexamen in Medizin ab. 1984 erwarb er den Facharzttitel in Allgemeiner Inneren Medizin und zwei Jahre später jenen in Rheumatologie. Nach einigen Jahren Spitaltätigkeit eröffnete er eine eigene Praxis, welche sich inzwischen zu einer Gruppenpraxis gewandelt hat. Parallel zu seiner ärztlichen Tätigkeit engagiert sich Herr Wiedersheim auch in der Standespolitik: Seit bald zwölf Jahren amtiert er als Präsident der Ärztegesellschaft des Kantons St. Gallen, Mitte 2007 wurde ihm die Leitung der Konferenz der Ostschweizer Ärztegesellschaften übertragen und seit 2012 ist er Co-Präsident der Konferenz der Kantonalen Ärztegesellschaften.

Neben diesen und weiteren standespolitischen Mandaten erwarb er 2003 den «Medical Manager» an der Privaten Hochschule Wirtschaft (PHW) Bern, St. Gallen, Zürich sowie ein Jahr später ebenfalls an der PHW einen Executive MBA. 


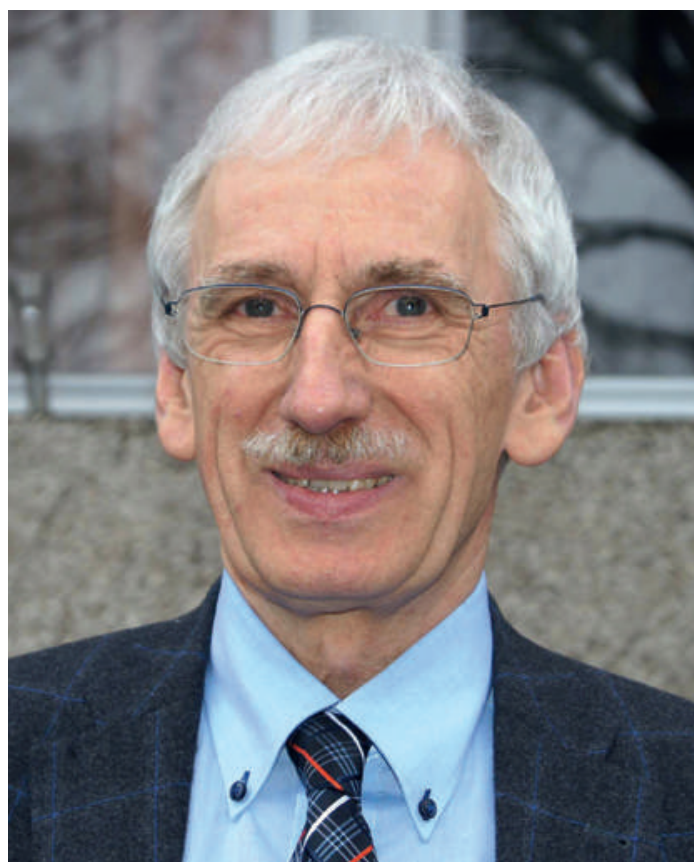

Zentrale Bedeutung hat für Peter Wiedersheim der Informationsaustausch über die Sprachgrenzen und die Führungsebenen hinaus.

Und wie gestalten Sie die Zusammenarbeit mit dem Zentralvorstand?

Nebst sehr guten und langjährigen persönlichen Kontakten werde ich wie mein Vorgänger an den Plenarsitzungen des Zentralvorstandes teilnehmen. Damit erhalte ich einen guten Einblick in die aktuel-
Im Jahr 2007 entstand die FMH-Delegiertenversammlung als Bindeglied zwischen dem Zentralvorstand und der Ärztekammer. Sie löste die rund 100 Personen umfassende Präsidentenkonferenz ab mit dem Ziel, ein kleineres und darum agileres Gremium zu schaffen für eine aktive Begleitung der Gesundheitspolitik. Mit ihren 33 Vertreterinnen und Vertretern der Dachverbände, welche von der Ärztekammer bestätigt werden, behandelt die Delegiertenversammlung als eigenständiges Organ alle zentralen gesundheits- und standespolitischen Aspekte und verabschiedet diese zuhanden der Ärztekammer. Ausserdem ist sie richtungsweisend für die Parolenfassung der FMH bei Volksabstimmungen sowie das Ergreifen einer Initiative oder eines Referendums.

Sie sind Co-Präsident der Konferenz der Kantonalen Ärztegesellschaften, Präsident der Ärztegesellschaft des Kantons St. Gallen und tragen somit verschiedene standespolitische Hüte. Entstehen da keine Interessenkonflikte bzw. sehen Sie Synergien für die Delegiertenversammlung?

Interessenkonflikte entstehen immer dann, wenn man unterschiedliche Ziele verfolgt. Mein erklärtes Ziel ist das Wohl respektive die Nutzenoptimierung für Patienten, egal welchen Hut ich trage. In unserer Arztpraxis heisst das konkret: «Ihre Gesundheit ist unser Ziel.» In der Standes- und Gesundheitspolitik gilt es, den optimalen Patientennutzen stets im Auge zu behalten - je besser wir diese Aufgabe lösen, desto besser geht es nicht nur uns, sondern allen!

\section{Aktuelle}

Forumthemen

Diskutieren Sie mit! Im Forum präsentieren wir regelmässig brisante Themen aus Politik, Ökonomie und Wissenschaft, die das Schweizer Gesundheitswesen betreffen. Bringen Sie Ihre Meinung ein oder kommentieren Sie die Äusserungen Ihrer Kolleginnen und Kollegen. Das Forum finden Sie unter: www.saez.ch/forum/

\section{«Der Austausch zwischen der Basis und den FMH-Gremien muss bidirektional optimiert werden.»}

len Themen und Arbeiten, auch wenn ich nicht immer in die Entscheidfindung einbezogen bin, die oft in den Klausursitzungen des Zentralvorstandes erfolgt. Durch meine vielfältigen standespolitischen Aufgaben ergeben sich auch zahlreiche Schnittstellen mit dem Zentralvorstand sowie dem Generalsekretariat der FMH. Die sehr gute Zusammenarbeit und der rege Informationsaustausch sind für meine Tätigkeit in der Delegiertenversammlung äusserst hilfreich.

Ich bin mir aber durchaus bewusst, dass die Delegiertenversammlung gemäss Geschäftsordnung auch ein Korrektiv-Organ ist im Sinne einer Gewaltentrennung zwischen der Exekutive und der Basis der Ärzteschaft.
Neben Ihren verschiedenen standespolitischen Ämtern sind Sie in einer Gemeinschaftspraxis als Facharzt für Innere Allgemeine Medizin sowie Rheumatologie tätig. Was ist Ihr Rezept für die Work-Life-Balance?

Ich habe gelernt, schon in kurzen Phasen abzuschalten und kann mich auch an kleinsten Selbstverständlichkeiten des Alltags freuen. Mein Fundament ist meine Familie, die nicht nur viel Verständnis für all meine Aktivitäten aufbringt, sondern mich auch nach Kräften unterstützt. Selbst mein 94-jähriger Vater sichtet noch immer täglich die Presse und schickt mir alle gesundheitspolitischen Artikel. Das Wichtigste ist jedoch, dass ich seit 35 Jahren in einem glücklichen Eheparadies leben darf. Meine Frau ist ein echter Engel - das verleiht wahrlich Flügel! 